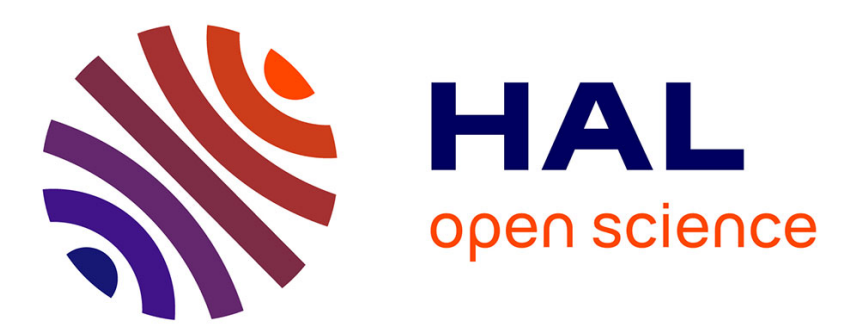

\title{
Reconfiguration of Assembly Lines under the Influence of High Product Variety in the Automotive Industry-A Decision Support System
}

Simon Altemeier, Marcel Helmdach, Achim Koberstein, Wilhelm Dangelmaier

\section{- To cite this version:}

Simon Altemeier, Marcel Helmdach, Achim Koberstein, Wilhelm Dangelmaier. Reconfiguration of Assembly Lines under the Influence of High Product Variety in the Automotive Industry-A Decision Support System. International Journal of Production Research, 2009, pp.1. 10.1080/00207540903252290 . hal-00540042

\section{HAL Id: hal-00540042 \\ https://hal.science/hal-00540042}

Submitted on 26 Nov 2010

HAL is a multi-disciplinary open access archive for the deposit and dissemination of scientific research documents, whether they are published or not. The documents may come from teaching and research institutions in France or abroad, or from public or private research centers.
L'archive ouverte pluridisciplinaire HAL, est destinée au dépôt et à la diffusion de documents scientifiques de niveau recherche, publiés ou non, émanant des établissements d'enseignement et de recherche français ou étrangers, des laboratoires publics ou privés. 


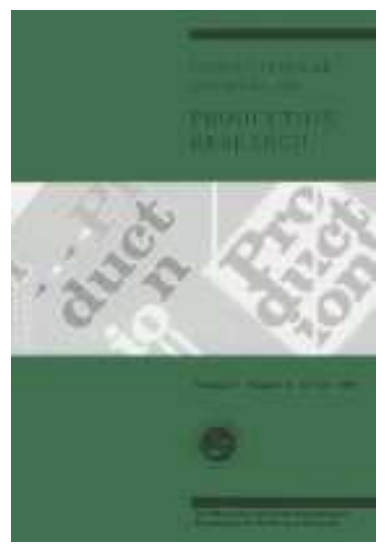

\section{Reconfiguration of Assembly Lines under the Influence of High Product Variety in the Automotive Industry-A Decision Support System}

\begin{tabular}{|r|l|}
\hline Journal: & International Journal of Production Research \\
\hline Manuscript ID: & TPRS-2009-IJPR-0167.R1 \\
\hline Manuscript Type: & Original Manuscript \\
\hline Author: & 27-Jul-2009 \\
\hline Complete List of Authors: & $\begin{array}{l}\text { Altemeier, Simon; Daimler AG, Group Research and Advanced } \\
\text { Engineering; Heinz-Nixdorf-Institut, CIM } \\
\text { Helmdach, Marcel; Daimler AG, Group Research and Advanced } \\
\text { Engineering } \\
\text { Koberstein, Achim; DSOR, Decision Support and Operations } \\
\text { Research Lab } \\
\text { Dangelmaier, Wilhelm; Heinz-Nixdorf-Institute University of } \\
\text { Paderborn, Business Computing, esp. CIM }\end{array}$ \\
\hline Keywords: & $\begin{array}{l}\text { ASSEMBLY LINE BALANCING, OPERATIONAL RESEARCH, } \\
\text { MANUFACTURING INFORMATION SYSTEMS, PRODUCTION } \\
\text { PLANNING, DECISION SUPPORT SYSTEMS }\end{array}$ \\
\hline Keywords (user): & ASSEMBLY LINE BALANCING, OPERATIONAL RESEARCH \\
\hline &
\end{tabular}

\section{ScholarONE \\ Manuscript Central}


International Journal of Production Research

Vol. 00, No. 00, 00 Month 200x, 1-22

\title{
ARTICLE \\ Reconfiguration of Assembly Lines under the Influence of High Product Variety in the Automotive Industry - A Decision Support System
}

Simon Altemeier ${ }^{a *}$, Marcel Helmdach $^{a}$, Achim Koberstein $^{b}$ and Wilhelm Dangelmaier ${ }^{c}$

${ }^{a}$ Daimler AG - Group Research and Advanced Engineering, Ulm, Germany; ${ }^{b}$ Decision Support and Operations Research Lab (DSOR-Lab) - University of Paderborn, Germany; ${ }^{c}$ Heinz-Nixdorf-Institute - University of Paderborn, Germany

(Received 00 Month 200x; final version received 00 Month 200x)

\begin{abstract}
In this paper we consider the mixed model assembly line reconfiguration problem in the context of auto production which is characterized by a make-to-order production process and a huge product variety. Starting from a given line balancing solution the goal is to minimize production costs in the short term for a largely known production program by reassigning and shifting tasks between workstations. We present a mathematical optimization model which aims at minimizing the costs incurred by overload situations, regular workers and reconfiguration measures. Due to the model's complexity, lack of data and acceptance issues it is hardly possible to fully automate the solution process in an industrial environment. Therefore, we present a decision support approach which consists of visualization components, new numerical indicators and an integrated heuristic optimization procedure to semi-automate the reconfiguration process. In particular, reconfiguration costs can be taken into account and no complete precedence graph is required. Finally, we show on the basis of two industrial case studies that our approach can be successfully applied in a practical environment where it was capable to drastically reduce the occurrence of overload situations.
\end{abstract}

Keywords: reconfiguration, assembly line balancing, automotive, product variety, decision support system

\section{Introduction}

In the automotive industry the number of product variants is extremely high and goes up to $10^{32}$ different models (Meyr 2004). Companies struggle with fluctuating customer demands, intensifying competition and shortening of product life cycles (Schuh 2006, Wiendahl and Gerst 2004). Due to the dynamic nature of demand forecasting proofs very difficult (Becker 2007, Gottschalk 2006). Therefore, regular adjustments of the assembly line setup are necessary (Bukchin et al. 2002). However, existing methods concentrate on the initial setup of an assembly line (Becker and Scholl 2006, Falkenauer 2005). Most of them are limited to an integral product architecture where average frequencies for the options are used and it is assumed that they are uniformly distributed. Other concepts take all possible variants into account but suggest a complete reassignment of the tasks to the workplaces from scratch. A practical implementation of these methods is very difficult as there is no reliable information about the production quantities of the variants over a whole product life cycle (Pohl 1996). Additionally, an adaption of the assembly line setup using these concepts would result in a complete reassignment of

\footnotetext{
*Corresponding author. Email: simon.altemeier@daimler.com
} 
all tasks for the next planning horizon. The necessary training of the workers, the changes in logistics and the costs to shift the tasks would outperform the gains by far. Furthermore, almost all concepts assume that a up to date precedence graph, exists. It causes a tremendous effort to put several thousands of tasks into relation to each other and keep them updated, and, to our best knowledge, no major car manufacturer has solved this problem so far. Even if there would be an efficient process to keep the data up to date, the precedence graph only includes the technical precedence relations; other complex restrictions, e.g., given by the layout of a line, space for parts and ergonomic aspects of task allocations, are not considered. Therefore, the existing models and methods for assembly line balancing are largely inappropriate for real life problems in the automotive industry (Falkenauer 2005).

In this work, we present a decision support approach, which uses exact information about the upcoming orders. The target is not only to reach a high workload on all the workplaces in average, but to minimize the occurrence of overload situations as well. Thereby, a minimization of floater operations and as little change in the existing balancing solution as possible is achieved.

The structure of this article is as follows. Firstly, the problem of reconfiguration is described in section 2, including a description of the considered assembly line system and a formal definition of the objective of the reconfiguration process. An overview of the related literature is given in section 3 . In section 4 , the problem is formalized and a complete mathematical model is presented. Our decision support approach is explained in section 5, including a tool for a what-if-analysis and a local search heuristic. In section 6 , results of two industrial case studies based on a software implementation of the approach are presented. Conclusions and issues for further research are discussed in section 7 .

\section{The reconfiguration problem in car production}

In the considered single line system, products are transported at a constant speed from station to station. New product items are placed on the conveyor belt at the interval of the cycle-time. Each station can contain several workplaces. Each workplace is assigned to a certain zone within the station. Workplaces in different zones can work in parallel on the workpiece. Only one workplace can be assigned to each zone. All stations are of the same length. This implies, given a constantly moving belt, that the working time available in one cycle is the same for all stations. For each product variant the set of tasks is given. Each of these tasks is assigned to exclusively one workplace. If multiple workers are assigned to one workplace, the capacity is simply multiplied by the number of workers. ${ }^{1}$

In contrast to the single-model case, where the cycle time is interpreted as an upper bound for the assigned workload, it corresponds to the average production rate. The workload may exceed the cycle time for particular variants, as long as other variants generate a workload sufficiently smaller. To compensate for this variation in workload, each workplace has a left and right drift area which enables the worker to leave the assigned station for a high workload variant. Even though drifting areas are available, overload situations can still occur. To deal with this problem there is an additional pool of high-skilled workers (floaters), which move to the affected workplaces and avoid overload situations by taking over one workpiece.

\footnotetext{
${ }^{1}$ In a practical scenario, so called two-cycle workers, being assigned to the same workplace, would alternately take a workpiece and work on it for two cycles. This implies, that these workers have to drift into the succeeding station. Becker and Scholl (2009) call this type of workplaces multiple-station workplaces.
} 
Page 3 of 22

\section{International Journal of Production Research}

Reconfiguration of Assembly Lines under the Influence of High Product Variety

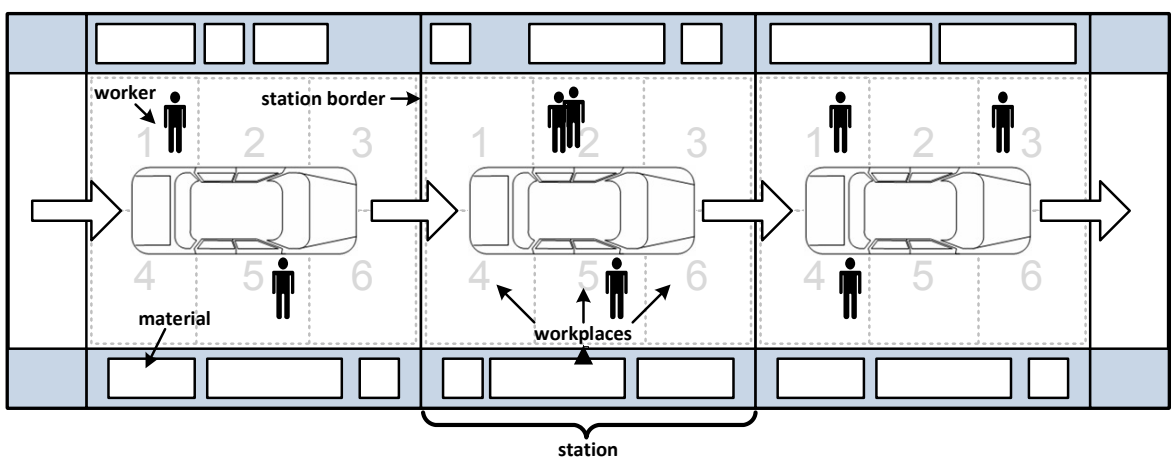

Figure 1.: Layout of the regarded assembly line system

The problem of reconfiguration is to find a new assignment of tasks that increases the efficiency. The planning horizon for the reconfiguration problem is defined by the time-span for which the demand is known with great certainty. Reconfiguration costs are incurred by every change of the existing assignment. Hence, the best solution is defined by the minimum sum of the reconfiguration costs and the costs of operation expected in the planning horizon.

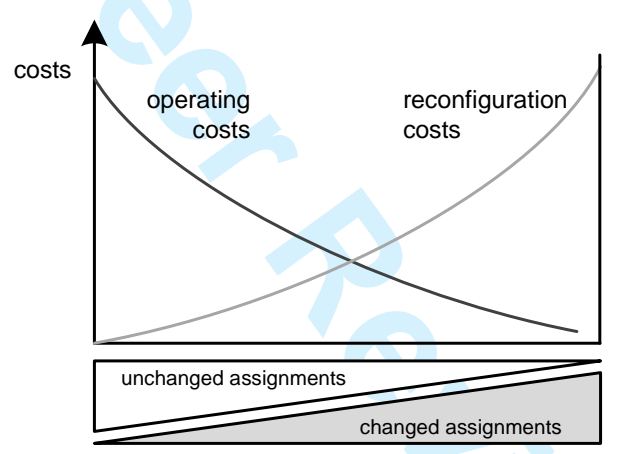

Figure 2.: Cost trade-off in a reconfiguration environment

Reconfiguration costs are typically incurred for:

- Retraining of workers

- Shifting of tools and storage racks

- Changing the delivery of parts

The operating costs consist of the costs for regular workers and the costs for floaters. While existing approaches concentrate on the number of necessary floater deployments only, the actual costs have to be derived from the number of necessary floaters that need to be hold ready for the deployments. Even if only a few number of floater deployments are necessary, numerous floaters are required if the situations happen at the same time. This number can be derived from the maximum number of concurrent overload situations. Floaters are high-skilled workers and are paid considerably more than a regular worker as they have to be able to do a lot more different tasks. Due to floater operations the probability for quality problems and the necessity of rework to fix failures increases as the usual production process is interrupted. Our practical experiences indicate that these additional costs highly correlate with the number of floater operations. 
3. Literature review

The assembly line balancing problem (ALBP) has been attracting the interest of numerous researchers in the fields of Operations Research and Industrial Engineering, since the first mathematical formulation was developed by Salveson (1955). Current overviews over the vast body of literature are given by Becker and Scholl (2006), Boysen et al. (2007a) and Boysen et al. (2008). Most of the literature deals with simplified versions of the problem, focusing on the assignment of tasks to stations while adhering a set of precedence constraints. According to widely accepted classification this basic problem setting is called the simple assembly line balancing problem (SALBP), if only one product variant is considered, and mixed model assembly line balancing problem (MALBP), if multiple product variants are considered. Further extensions of the problem setting like parallel lines, multiple workers per station etc. are subsumed by the term general assembly line balancing problem (GALBP). Objectives of optimization are typically the minimization of the number of stations (equivalent to minimization of idle times), the minimization of the cycle time or both (maximization of line efficiency). More recent models also try to considers costs of production explicitly. Successful solution approaches typically apply branch-and-bound methods for the SALBP (cf. Scholl and Klein (1997), Sprecher (1999), Sprecher (2003), Erel et al. (2005)) and heuristics and meta-heuristics for the more complex MALBP and GALBP (see e.g. McMullen and Frazier (1998), Chiang (1998), Kim et al. (2000) and Vilarinho and Simaria (2002)).

Merengo et al. (1999) point out that solution methods for the MALBP always have to take into account the actual production mix. The authors propose a balancing and sequencing approach which aims at minimizing the number of stations, the rate of incomplete jobs and reduce work-in-process. However, they assume that reconfiguration costs are negligible and a complete reassignment of tasks is possible.

Bock et al. (2006) present a model and a solution approach for the simultaneous reassignment of tasks and the adaption of the production sequence in the presence of disruptions during the assembly line operations. Some tasks can be fixed to stations. However, the majority of the remaining tasks can be reassigned at no additional costs.

Domschke et al. (1996) describe an assembly line balancing approach for medium term planning which focuses on the simultaneous minimization of fluctuations within the assembly line besides the traditional goals minimization of the number of stations and the cycle time. Some examples are given which illustrate the avoidance of work-overload situations by solving a succeeding sequencing problem. As the preceding approaches the authors utterly disregard existing assembly lines and propose a complete reassignment of tasks to stations.

Becker and Scholl (2009) extend the basic ALBP to the case of multiple workplaces per station where tasks can be carried out in parallel. They develop an integer linear programming model and a branch-andbound type solution procedure. Computational results are presented which indicate that small to medium size instances can be solved to optimality and solutions of high quality can be found for larger instances by using the branch-and-bound procedure in a heuristic manner. Model and solution procedure aim at generating a solution from scratch, no reconfiguration costs are considered. As in many other similar approaches the existence of complete precedence graph is a necessary precondition.

The term reconfiguration problem has been used in slightly different senses in the ALB literature. Some authors denote the problem of physically redesigning 


\section{Formalization of the problem}

In this section we develop a mathematical model for the reconfiguration problem. It will be based on the notation given in Table 1. An exact evaluation of a reconfiguration solution is only possible if the sequence in which the products will be put onto the line is known. Due to different planning horizons the reconfiguration and the sequencing problem cannot be solved simultaneously (see Boysen et al. (2007b)). Since the exact production sequence cannot be known at the execution of the reconfiguration planning task, the sequence dependent part of the objective function, consisting of the costs for floaters, has to be replaced by a sequence independent criterion that anticipates the results of the succeeding sequencing solution (as indicated in equation (1)).

$$
\begin{aligned}
\operatorname{Min} \quad \mathcal{C}^{\text {Total }}= & \overbrace{\mathcal{C}^{f l t} \cdot f l t^{\text {max }}+\mathcal{C}^{\text {Rework }} \cdot f l t^{\text {Operations }}}^{\text {To be substituted by an anticipating criterion }} \\
& \sum_{w p \in \mathcal{W P}} \mathcal{C}^{\text {Worker }} \cdot w_{w p}+\sum_{w p \in \mathcal{W P}} \sum_{i \in \mathcal{I}} \mathcal{C}_{i, w p}^{\text {Reconf }} \cdot v_{i, w p}
\end{aligned}
$$

In the context of MALBP models some objectives have been proposed which anticipate the results of the succeeding sequencing solution (see Emde et al. (2008) for a recent comprehensive study). Domschke et al. (1996) show that the sum of the positive deviations from the cycle time is a good criterion for this purpose, which was recently confirmed 
Table 1.: Notation

by Emde et $\boldsymbol{a l}$. (2008). In equations (2) to (12) we give a complete mathematical 
model for the reconfiguration problem.

$$
\begin{aligned}
& \text { Min } \quad \mathcal{C}^{\text {Total }}=\mathcal{C}^{\text {LinPos }} \cdot \sum_{w p \in \mathcal{W} \mathcal{P}} \sum_{p \in \mathcal{P}} \max \left\{0, d_{p, w p}-c \cdot w_{w p}\right\}+ \\
& \sum_{w p \in \mathcal{W P}} \mathcal{C}^{\text {Worker }} \cdot w_{w p}+\sum_{w p \in \mathcal{W} \mathcal{P}} \sum_{i \in \mathcal{I}} \mathcal{C}_{i, w p}^{\text {Reconf }} \cdot v_{i, w p}
\end{aligned}
$$

s.t.

$$
\sum_{w p \in \mathcal{W P}} x_{i, w p}=1
$$

$v_{i, w p} \geq x_{i, w p}-z_{i, w p}$ $\forall w p \in \mathcal{W P}, i \in \mathcal{I}$

$$
\sum_{w p \in \mathcal{W P}} s t_{w p} \cdot x_{i, w p} \leq \sum_{w p \in \mathcal{W} \mathcal{P}} s t_{w p} \cdot x_{j, w p}
$$$$
\forall(i, j) \in \mathcal{P} \mathcal{R E C}
$$

$$
\begin{aligned}
& \sum_{i \in \mathcal{I}} \text { freq }_{i} \cdot \tau_{i} \cdot x_{i, w p} \leq c \cdot w_{w p} \\
& d_{p, w p}=\sum_{i \in \mathcal{I}} \tau_{i} \cdot b p_{p, i} \cdot x_{i, w p}
\end{aligned}
$$$$
\forall w p \in \mathcal{W P}
$$

$\sum_{i \in \mathcal{I}} n s_{i} \cdot x_{i, w p} \leq a s_{w p}$

$\forall \quad w p \in \mathcal{W P}$

$$
x_{i, w p}=0
$$

$\forall w p \in \mathcal{W P}, i \in \mathcal{I}:$

$$
\operatorname{pos}_{w p} \notin P O S_{i}
$$

$$
x_{i, w p} \in\{0,1\}
$$

$\forall w p \in \mathcal{W P}, i \in \mathcal{I}$

$$
v_{i, w p} \in\{0,1\}
$$

$$
w_{w p} \geq 0 \text { and integer }
$$

In the objective function (2) the sequence dependent part of equation (1) has been replaced by the total overload-time for our case of multiple workers per workplace (this part of the objective is a multi-manning version of objective 3 in Domschke et al. (1996)). Equation set (3) ensures that each task is assigned to exactly one workplace. Equation set (4) accounts for changes in the assignments compared to an incumbent balancing solution. These changes induce reconfiguration costs in the objective function. Precedence relationships are preserved by equation set (5). It is assumed that there always exists a feasible schedule within each station. While this assumption may not be true in theory, it usually is in practical environments. The number of workers per workplace is derived from the average workload in equation set (6). This assures that, overall, a minimum number 
of workers per workplace is assigned and sustained overload situations are avoided. The total execution time for a product variant is computed in equation set (7). Equation set (8) guarantees that the available space of a workplace is not exceeded by the space required by the assigned tasks. The workpiece can be in different positions in each workplace, e.g. lower, upper, tilted or upside down position. Certain tasks can only be executed if the workpiece is in a certain position, which makes certain assignments ineligible in equation set (9).

Exact or heuristical solution methods could be developed to solve the above model. However, deploying it directly in a practical environment would give rise to a number of issues which cannot easily be resolved. They include:

- The model requires a huge amount of data, which can be difficult to obtain. For instance, $|\mathcal{I}| \cdot(|\mathcal{W} \mathcal{P}|-1)$ many cost coefficients for the reconfiguration costs have to be estimated in advance. Additionally, these costs can actually depend on the task assignment. So after each shift of a task, a reevaluation of all costs from and to the two concerned workplaces would be required.

- Obtaining a valid and comprehensive precedence graph poses a big problem in car manufacturing. One obstacle is the sheer number of different tasks, another the constant change in the design of the products. To our best knowledge, no major car manufacturer has managed to use the concept of a precedence graph so far, although a lot of R\&D efforts have been undertaken to develop the necessary methods and tools (an interesting new option based methodology has recently been proposed by Boysen et al. (2009)).

- In a real world manufacturing line numerous additional practical restrictions come up. It is virtually impossible to consider all of them in the above or a similar model.

Unfortunately, hardly any model based approach from theory has been implemented as a practical solution being used in the industry so far (see Falkenauer (2005) for further discussion). Therefore we propose a concept and an implementation of a decision support system that is based on the above reconfiguration model but integrates the planner with his knowledge into the decision process.

\section{A decision support approach}

The discipline of decision support has been investigated since many years and Decision Support Systems (DSS) have been developed for many different purposes. A general architecture for DSS was proposed by Turban and Aronson (1998) ${ }^{1}$, which will provide the basis for our system:

- In the Analysis-Phase data is processed, aggregated and different key indicators are generated, which characterize the problem. The main intention is to identify the main issues and help the user to focus on these.

- The purpose of the Design-Phase is to help the user to design alternative planning solutions.

- In the Decision/Selection-Phase different planning solutions are evaluated and the user is supported in deciding for the best solution.

- The intention of the Implementation-Phase is to support the planner at implementing the chosen planning solution. Also, fall-back strategies are provided if certain parts of the solution fail to be implemented.

\footnotetext{
${ }^{1}$ see also (Hotman 2006, p.31) and (Smith 1998, p.6)
} 


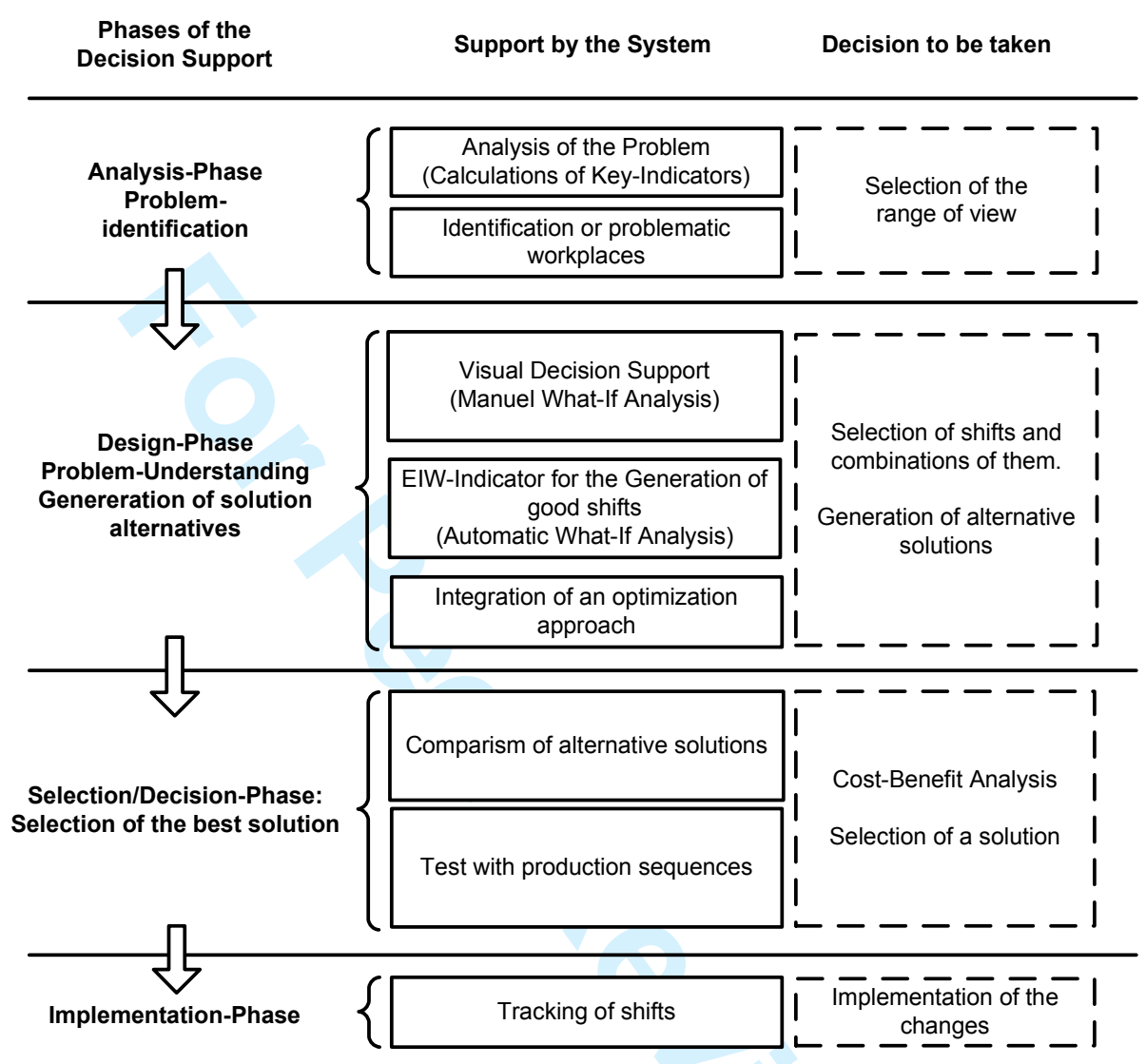

Figure 3.: Phases of the Decision Support System

\subsection{Analysis-Phase}

The reconfiguration process is triggered as soon as the orders for the next planning period are fixed. Due to the new production program, the workloads $d_{p, w p}$ on the workplaces change and a reassignment may become necessary. To calculate the new workloads, the parameter $b p_{p, i}$ has to be recomputed first to comply with the new production program. This is a non-standard operation which can be conducted using the bill of material in conjunction with the bill of processes for each product variant to be produced. The existing planning solution is set as the starting point for the new balancing solution:

$$
x_{i, w p}=z_{i, w p} \quad \forall i \in \mathcal{I}, w p \in \mathcal{W} \mathcal{P}
$$


Table 2.: Additional notation

The new workloads can now be computed according to equation set (7). Also, task frequencies and average workloads need to be recomputed:

$$
\begin{aligned}
\text { freq }_{i} & =\frac{1}{|P|} \sum_{p \in \mathcal{P}} b p_{p, i} \\
\mu_{w p} & =\sum_{i \in \mathcal{I}} \tau_{i} \cdot \text { freq }_{i} \cdot x_{i, w p}
\end{aligned}
$$

Three types of actions to react to the changes can be distinguished:

- Repair: Resolve overloads in average workloads.

- Compact: Minimize the number of workers by clearing workplaces.

- Anticipate: Minimize number of floater operations by decreasing the number of overload-situations.

In the following, we present key indicators for each of these actions, which support the planner in identifying critical workplaces ${ }^{1}$.

Repair

A small increase in the frequencies of tasks assigned to a workplace can cause the average workload to exceed its capacity (cycle time times number of regular ${ }^{1}$ This is comparable to the "'Issue Detection"' process, defined by (Smith 1998, S.44) and the process of
analysis in Turban and Aronson (1998) 
workers). In such a case, an additional worker, of course having a very low average workload, had to be assigned. To enable the planner to quickly recognize such situations we compute the positive difference between the new number and the old number of assigned workers on a workplace:

$$
\Delta w_{w p}=\max \left\{0,\left\lceil\frac{\sum_{i \in \mathcal{I}} \tau_{i} \cdot f r e q_{i}^{\text {new }} \cdot x_{i, w p}}{c}\right\rceil-\left\lceil\frac{\sum_{i \in \mathcal{I}} \tau_{i} \cdot f r e q_{i}^{\text {old }} \cdot z_{i, w p}}{c}\right\rceil\right\}
$$

The opposite case, i.e, a reduction of the number of assigned workers due to the changed production program, is very unlikely, but can happen if a major difference in the production program exists. However, a direct need for action is not to be derived from this situation.

\section{Compact}

Compacting is the process of emptying underemployed workplaces by shifting tasks to nearby workplaces with free capacity. However, it only makes sense to compact the assembly-system if a complete worker can be saved and the reconfiguration costs do not way out the gains. Tasks have to be shifted away from a workplace, until the number of necessary workers can be decreased with respect to the average workload. Enough space for those tasks on the other workplaces is necessary to make such a compact operation possible.

In a first step the workplace with the highest potential for a compact operation is selected by using the indicator presented in equation (17). This is the workplace with the greatest relative gap between the existing capacity $w_{w p} \cdot c$ and the average workload $\mu_{w p}$.

$$
\Delta \mu_{w p}=1-\frac{\mu_{w p}}{c \cdot w_{w p}}
$$

The least amount of workload that needs to be shifted away from a candidate workplace to make a compact operation possible can be computed as follows:

$$
\epsilon_{w p}=\mu_{w p}-\left(c \cdot\left(w_{w p}-1\right)\right)
$$

Furthermore, enough idle time on the alternative workplaces has to exist ${ }^{1}$. Let $w p_{1}$ be the candidate workplace, then the following needs to hold true:

$$
\epsilon_{w p_{1}} \leq \sum_{w p_{2} \in \mathcal{W} \mathcal{P}: w p_{1} \neq w p_{2}} \Delta \mu_{w p_{2}}
$$

For a final decision, whether a compact-process makes sense or not, the planner has to compare the costs for shifting the tasks with the costs for the worker to be saved.

\section{Anticipate}

The anticipation process attempts to minimize the required floater operations in the production process. For this purpose, workplaces with a high risk to generate

\footnotetext{
${ }^{1}$ This is a necessary but not a sufficient condition. Further conditions are given by the precedence graph, the ratio between the costs for the shifting operations and the costs for workers that can be saved.
} 
floater operations have to be identified. We use the linear overload of a workplace, denoted by $\Delta_{w p}^{\operatorname{LinPos}}$, as a key indicator to identify such workplaces:

$$
\Delta_{w p}^{\operatorname{LinPos}}=\sum_{p \in \mathcal{P}} \max \left\{0, d_{p, w p}-c \cdot w_{w p}\right\}
$$

Even though it seams obvious to use this indicator, a large workload gap $\Delta \mu_{w p}$ may give the workplace enough flexibility to cope with the overload situations and the potential to generate floater operations might not be as high as assumed. Therefore, we introduce an indicator measuring the ratio between the linear overload and the workload gap:

$$
\rho_{w p}^{\operatorname{LinPos}}=\frac{\Delta_{w p}^{\operatorname{LinPos}}}{\Delta \mu_{w p}}
$$

Additionally, a squared version of the indicator defined in (20) can be used in extreme overload situations:

$$
\Delta_{w p}^{\text {QuadPos }}=\sum_{p \in \mathcal{P}} \begin{cases}\left(d_{p, w p}-c \cdot w_{w p}\right)^{2}, & \text { if } d_{p, w p} \geq c \cdot w_{w p} \\ 0, & \text { otherwise }\end{cases}
$$

\section{Selection of the Range of View}

The proposed key-indicators support the selection of candidate workplaces for the three steps repair, compact and anticipate. For the practicability of a decision support system, it is necessary to limit the complexity by focussing on a few workplaces. This guarantees that the planner is not flooded with information and that algorithms have to cope with less complexity.

After the selection of a problematic source workplace, alternative target workplaces have to be selected where tasks can be shifted to. The target workplaces have to fulfill several requirements regarding the position of the workpiece, the workload gap and the storage space.

The closer potential target workplaces are to the source workplace, the higher is the probability to find shifts that fulfill the precedence restrictions. If a task is shifted backwards in the production process, no tasks between the source and the target workplace may have a precedence relation with it. The same is true for shift in the forward direction of the production process. If a task has a precedence relation to the shifted task, it has to be checked if it can be shifted as well. To minimize these complex situations source and target workplaces should be as close as possible to each other. ${ }^{1}$

Regarding the ergonomics and reduction of operation times it makes sense to look at workplaces being assigned to the same zones of their stations. If tasks are shifted between workplaces assigned to different zones the worker might have to do additional movements and therefore increasing operation times can be the result. Therefore a selection of workplaces being assigned to the same zones should be prioritized. A complete restriction to these constellations would be misleading, as flexibility in the solution process would be lost.

The ultimate decision about the selection of workplaces can only be done by the planner himself as this is dependent from the potential shifting costs and training

\footnotetext{
${ }^{1}$ This usually minimizes costs for retraining the workers as well, as most workers are able to do tasks of
} neighbor workplaces, too. 


\subsection{Design-Phase}

In the following, ways to improve the understanding of the problem are presented and means to support the search process for better alternative balancing solutions are developed.

\subsubsection{Visual Decision Support}

Supplementary to the average workload of a workplace, all processing times associated with the production program of the next period are presented in a histogram. In these histograms the y-axis shows intervals of processing times. Bars, stretching in $\mathrm{x}$-direction, represent the frequency with which certain processing times occur (number of products requiring a processing time within the interval). The user can define the sizes of the intervals.

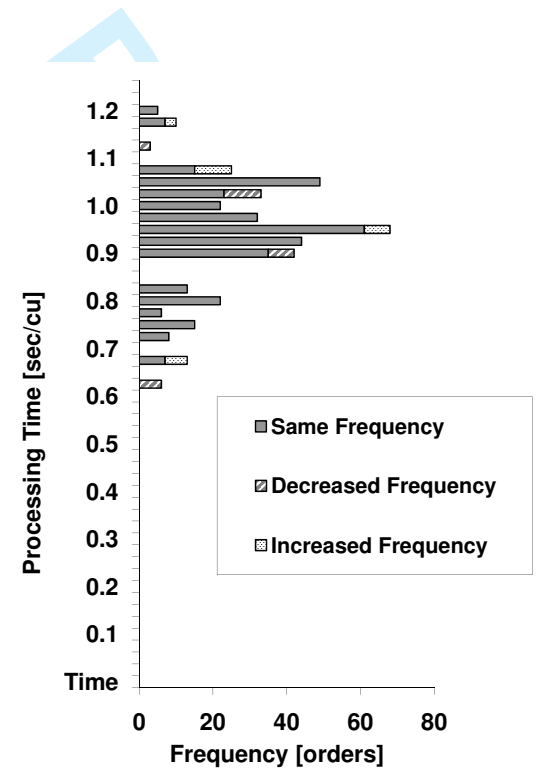

Figure 4.: Changes in the histogram after a process shift.

Furthermore, the decision support approach enables the user to see the changes in the histograms after shifting a process to another workplace (see Figure 4) by displaying the bars in different colours and shapes. An increase in the number of products associated with a processing time interval is shown by dashed bar. A decrease is illustrated by a dotted bar. It is possible to show the effect of just one shift or the summarized effect of numerous shifts at once. As a further aid, the user can see the impact of shifting a task to all alternative workplaces at a glance, prior to the actual shift.

Besides the graphical tools, the decision support approach displays the important key figures, including the average workload, the workload gap and the linear as well as the squared overtime for each workplace. These figures are recalculated after a shift is done. To support the anticipation process, the ratios between the linear and squared overload times and the workload gap are displayed and updated as well. 


\subsubsection{The EIW-Indicator}

As described earlier, it is important to minimize overload situations in order to minimize floater operations. Thus it is especially important to find shifts which minimize the sum of the linear overtime. The described means can be used to find those shifts manually, but due to the amount of processes in a practical environment further support is necessary. Therefore, we developed a special indicator, which aims at facilitating the evaluation of the potential of a shift to actually contribute the overtime reduction.

The basic idea is the following: consider a task $i$ that is required by a workpiece $p \in \mathcal{P}$, which induces a high workload on a certain workplace $w p \in \mathcal{W P}$. In the histogram in Figure 5 the task would contribute to the higher bars on the y-axis. It would be highly desirable to shift task $i$ to another workplace because such a shift had a high probability of reducing overload on workplace $w p$. At the same time, a target workplace has to be found where task $i$ does not create new or additional overload. That is likely the case if, on the target workplace, task $i$ contributes only to the lower bars of the histogram. The measure effect in workplace $(E I W)$ indicates the relative workload situation on a certain workplace when a single task is carried out. It is defined as follows $(|\cdot|$ denotes the cardinality operator):

$$
E I W_{i}^{w p}=\frac{1}{w_{w p} \cdot c \cdot\left|\mathcal{P}_{i}\right|} \sum_{p \in \mathcal{P}_{i}} d_{p, w p}
$$

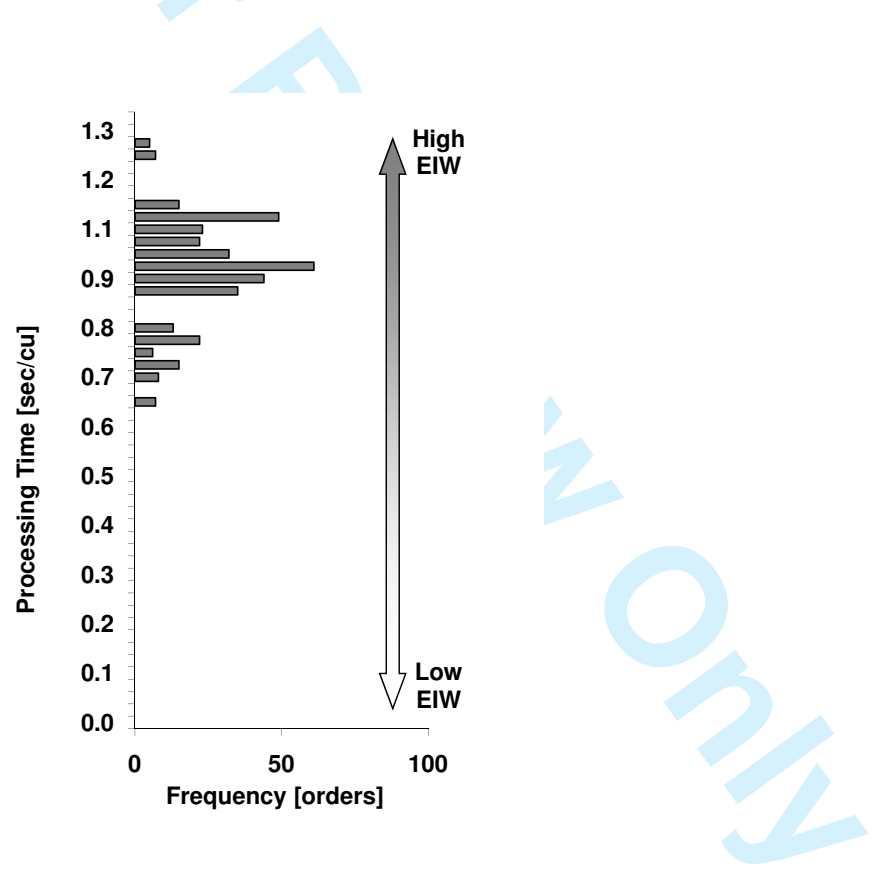

Figure 5.: Interpretation of the EIW

Tasks having a rather high EIW, especially with an average summarized workload above the cycle time (EIW > 1) should be moved away from their workplaces to reduce over-cycle time. To reduce the sum of the linear overtime of all regarded workplaces, the EIW of a task has to be lower on the target workplace. This assures that the overload is not just shifted to another workplace but eliminated. If there are several candidate workplaces for one process, the workplace with the 
lowest EIW should be selected. Before shifting a task from one workplace to another it should always be ensured that the remaining average idle time on the new workplace does become to small. One way of interpreting the EIW is that tasks required by the same workpiece should be distributed among different workplaces (better: stations) where possible, whereas tasks assigned to one workplace should ideally be required by different workpieces (we call these tasks complementary).

\subsubsection{Integration of an optimization approach}

The manual reconfiguration part of the decision support approach is complemented by a local search heuristic. It increases the speed of the reconfiguration process, as numerous shifts are evaluated successively. All diagrams and indicators we presented up to now can only support the selection of the "next" shift whereas the local search heuristic finds a good combination of shifts to reach a better objective value. Based on an existing solution new solution proposals are calculated. Following the search process the user still has to be able to check the proposal and decide whether all shifts in the solution are feasible and worth realizing. Typically, he will evaluate the reconfiguration costs for each shift in the sequence and decides to accept it or omit it. Therefore, the proposals generated by the algorithm should not include to many shifts, as the overview of the user would be lost otherwise. Thereby a higher acceptance of the system is achieved in a practical environment. The heuristic searches a limited neighborhood of an existing solution and presents the best found sequences to the user. The user can define how many solutions should be presented and how extensive the search should be.

At first, the initial solution has to be evaluated. The user can choose between two different objective functions: the linear overload and the squared overload. The heuristic will generate proposals which reduce either the one or the other. After this first calculation, the neighborhood of the initial solution, given by all possible shifts of one process to other workplaces, is evaluated. Shifts can be invalid due to the following restrictions:

(1) Precedence restrictions: As explained above, many manufacturers only have an incomplete precedence graph or even none at all. For this reason, the decision support system offers the possibility to use an existing (partial) graph and to change, add or deactivate precedence restrictions.

(2) Workplace limits: Workplace limits can be defined to keep the average workload on a workplace in limits. These limits are entered as percentage values for the average workload of a certain workplace. Any shift that leads to a limit violation is invalid. For instance, an upper limit of $99 \%$ means, that the average workload of a certain workplace must not become higher than $99 \%$ by newly proposed shifts.

(3) Permitted workplaces: Each process gets a list of allowed workplaces, e.g., to make sure that it is only assigned to workplaces where the product is in the right working position. Each shift that moves a process to a workplace that is not in its list is invalid.

(4) Fixed processes: Some processes must remain at a specific workplace due to special machinery or specially qualified workers (cf. Scholl et al. (2009)). As a result, the original workplace of each of these processes is the only allowed workplace. Therefore, shifts including these processes are neglected.

The neighborhood consists of all solutions that can be obtained from the original solution by doing one valid shift. To evaluate the neighborhood the overload, linear or squared, has to be calculated. However, these magnitudes need not to be cal- 


\section{International Journal of Production Research}

culated from scratch for each solution. It is sufficient to determine the differences induced by the current shift (cf. Equations (24) and (25)). This is extremely important in a practical environment, as the responsiveness of the system is crucial for a high acceptance by the users.

$$
\Delta_{i}^{\text {LinPos }^{-}}=\frac{1}{\left|\mathcal{P}_{i}\right|} \cdot \sum_{p \in \mathcal{P}_{i}}\left(\max \left\{0,\left(d_{p, w p}-\tau_{i}\right)-c \cdot w_{w p}\right\}-\max \left\{0, d_{p, w p}-c \cdot w_{w p}\right\}\right)
$$

where $w p \in \mathcal{W P}$ such that $x_{i, w p}=1$,

$$
\Delta_{i}^{\text {LinPos }}{ }^{+}=\frac{1}{\left|\mathcal{P}_{i}\right|} \cdot \sum_{p \in \mathcal{P}_{i}}\left(\max \left\{0,\left(d_{p, w p}+\tau_{i}\right)-c \cdot w_{w p}\right\}-\max \left\{0, d_{p, w p}-c \cdot w_{w p}\right\}\right)
$$

where $w p \in \mathcal{W P}$ such that $z_{i, w p}=1$.

Finally, the two values $\Delta_{i}^{\operatorname{LinPos}^{-}}$and $\Delta_{i}^{\mathrm{LinPos}^{+}}$are summed up. The lower the value is, the higher is the reduction of overcycle time and the better the solution. The best $n$ solutions are selected and saved. This defines how extensive the search is going to be. Each of these solutions differs from the original solution by one shift. Therefore, we call them "solutions of the first stage". Each saved solution is starting point for a new neighborhood analysis. Again, the best solutions are selected and saved. Thereby, solutions of the second stage are created. This procedure is repeated until a certain predefined stage $m$ is reached (cf. Figure 6).

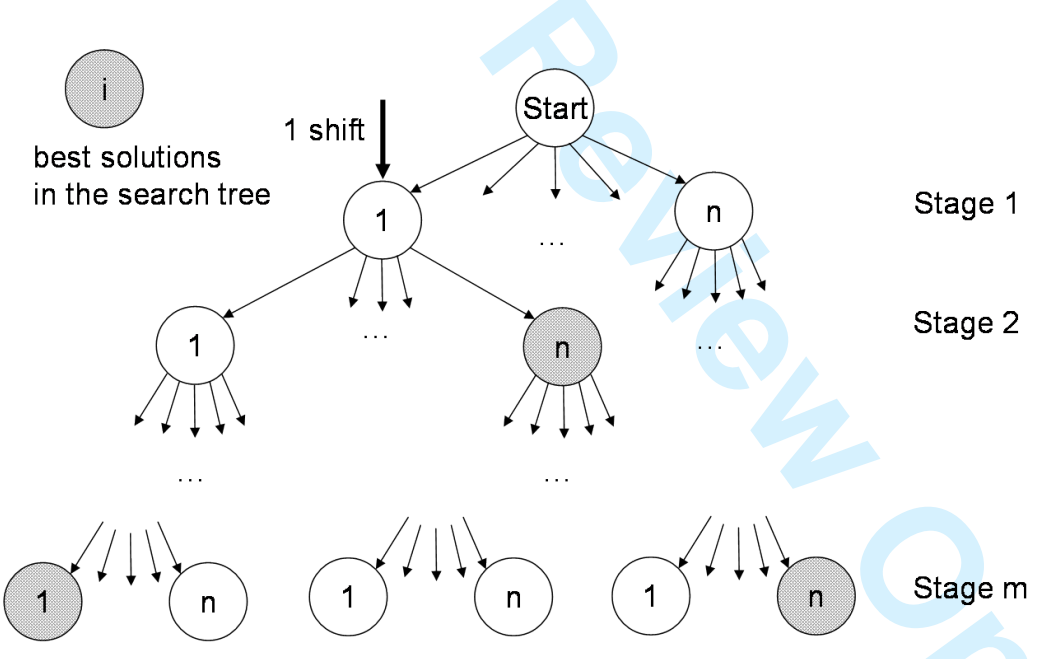

Figure 6.: Search tree for $\mathrm{m}$ stages and $\mathrm{n}$ solutions per stage

The best $k$ non-identical solutions of the search tree are stored ${ }^{1}$. For the user's evaluation process, the solutions are presented using the histograms described earlier. The different shapes and colours of the bars help the user to see the differences between the new and the original solution. The updated key figures are displayed as well.

\footnotetext{
${ }^{1}$ There may be identical solutions in the search tree because of different shift sequences.
} 


\section{Page 17 of 22 \\ International Journal of Production Research IJPR}

Reconfiguration of Assembly Lines under the Influence of High Product Variety

\subsection{Selection/Decision-Phase}

In this phase the planner compares the key-figures resulting from an implementation of the candidate solutions with the resulting reconfiguration costs. Given a task assignment, the real workloads, floater deployments, occurrences of drifting, etc. can only be simulated based on a or a set of given production sequence(s). However, this computation should be seen as an additional test of a planning solution but not as the primary evaluation method because it can be very time consuming and the actual production sequence cannot be foreseen at the time of reconfiguration.

\subsection{Implementation-Phase}

In the Implementation Phase, the selected solution is implemented at the assembly line. The necessary shifts to get to the new planning solution can be derived from the difference between the new selected assignment solution and the existing setup. During the actual implementation, problems may come up, the planner did not think about before. Therefore, the planner must have the option to undo a shift he had selected. A loop back to the decision phase is always possible to find another alternative shift or stick to the remaining solution.

\section{Case Study and Results}

In this section, we evaluate our decision support approach on the basis of a case study from a major car manufacturer. In two special workshops, production planners conducted a reconfiguration for part of an assembly line consisting of several workplaces. We evaluate and compare the results of the reconfiguration with and without the decision support system.

Before the workshops, the assembly lines had already been reconfigured by the planners. The only tool to help them was a program updating the average workload after each shift. As a result, the planners could already resolve overloads in average workloads (repair) and they could minimize the number of workers by clearing workplaces as far as possible (compact). However, they were not able to consider the number of overload situations (anticipate). In two workshops, the planners applied our decision support approach and software tool to decrease the positive deviation on selected workplaces.

To evaluate the impact of the reconfiguration in the most realistic way, an assembly line simulation tool was developed, which calculates actual worker positions for a selected part of the assembly line or the whole line. A given car sequence is used to simulate start and end working times for each workplace and each car. If a worker is not able to finish all his tasks at a car within the given cycle time, he will not only drift out of his own station, but will also start his work on the following car later. Additionally, he can only drift a certain distance out of his station. Otherwise an assistance worker (floater) is assigned who helps him to finish his tasks in time. The start working time at a workplace is not only determined by the drift of the worker on his workplace but also by workers of preceding workplaces. If a worker drifts into the next station, he can hinder another worker. These interactions between the workplaces are taken into account as well.

We will now illustrate the approach by looking at a pair of workplaces from our case study. The tasks assigned to these workplaces contain operations at the interior of the car. The workload at the workplaces is very fluctuating as can be seen in Figure 7 . The average workload at workplace 1 is $86 \%$ and $84 \%$ at workplace 2 . 
Every indicator is measured relative to the cycle unit [cu] which is the cycle time. At workplace 1, roughly 600 of about 5,000 cars require a workload higher than the cycle time. As a result, each car has an average linear overload-time of $0.015 \frac{\mathrm{sec}}{\mathrm{cu}}$ and an average square overload-time of $0.160 \frac{\mathrm{sec}^{2}}{\mathrm{cu}}$. At workplace 2, there are even 2,300 cars with more workload than the cycle time. As a consequence, the average linear overload-time is $0.045 \frac{\mathrm{sec}}{\mathrm{cu}}$ and the square overload-time is $0.370 \frac{\mathrm{sec}^{2}}{\mathrm{cu}}$.
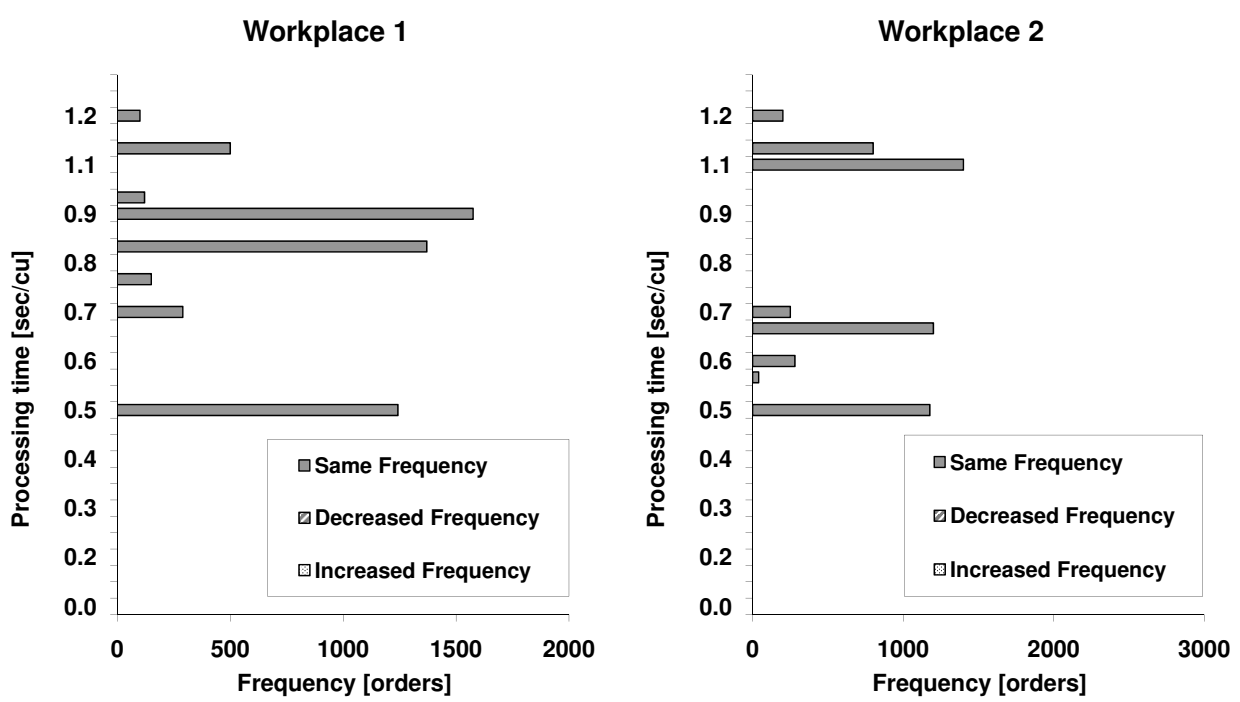

Figure 7.: Two workplaces considered for a reconfiguration

Now, a task has to be found that decreases the linear overload-times. Therefore, the EIW ${ }^{1}$ for all tasks of the two workplaces is calculated for their original and the second workplace. As a result, one task was found whose EIW at workplace 1, its original workplace, is 1.013 and 0.856 at workplace 2. Consequently, shifting this task will decrease some overload-time at workplace 1 and add less or non at workplace 2. Furthermore, the shift of this task from workplace 1 to workplace 2 is valid. The task needs $0.215 \frac{\mathrm{sec}}{\mathrm{cu}}$ and is required in $42 \%$ of the cars. By shifting it to workplace 2 , all overload-time at workplace 1 is removed. At the same time, no overload-time is added to workplace 2 . The details of the shift can be seen in the histograms of Figure 8.

After performing the shift, the average workload on both workplaces has changed. The average workload at workplace 2 has increased to $93 \%$ and, at the same time, the workload at workplace 2 has decreased to $77 \%$. To rebalance the workplaces, a shift from workplace 2 to workplace 1 is needed. A candidate for such a shift is a task of $0,113 \frac{\mathrm{sec}}{\mathrm{cu}}$ that is required in $23 \%$. Its EIW at workplace 2 is 1.103 and 0.767 at workplace 1 and the shift is valid. Moving this task to workplace 1 leads to a decrease of the overload-time at workplace 2 and does not generate new overload-time at workplace 1 (cf. Figure 9).

For the planners the reconfiguration of the two regarded workplaces seemed optimal. However, it was possible to decrease the linear overload-time from $0.060 \frac{\mathrm{sec}}{\mathrm{cu}}$ to $0.024 \frac{s e c}{c u}$ with only two further shifts. This means a decrease of $60 \%$ of linear overload-time. Moreover, the square overload-time was decreased to even $72 \%$. At the same time, the resulting average workload was changed only slightly from $86 \%$ to $80 \%$ on workplace 1 and from $84 \%$ to $90 \%$ on workplace 2 .

\footnotetext{
${ }^{1}$ see also chapter 5.2 .2
} 

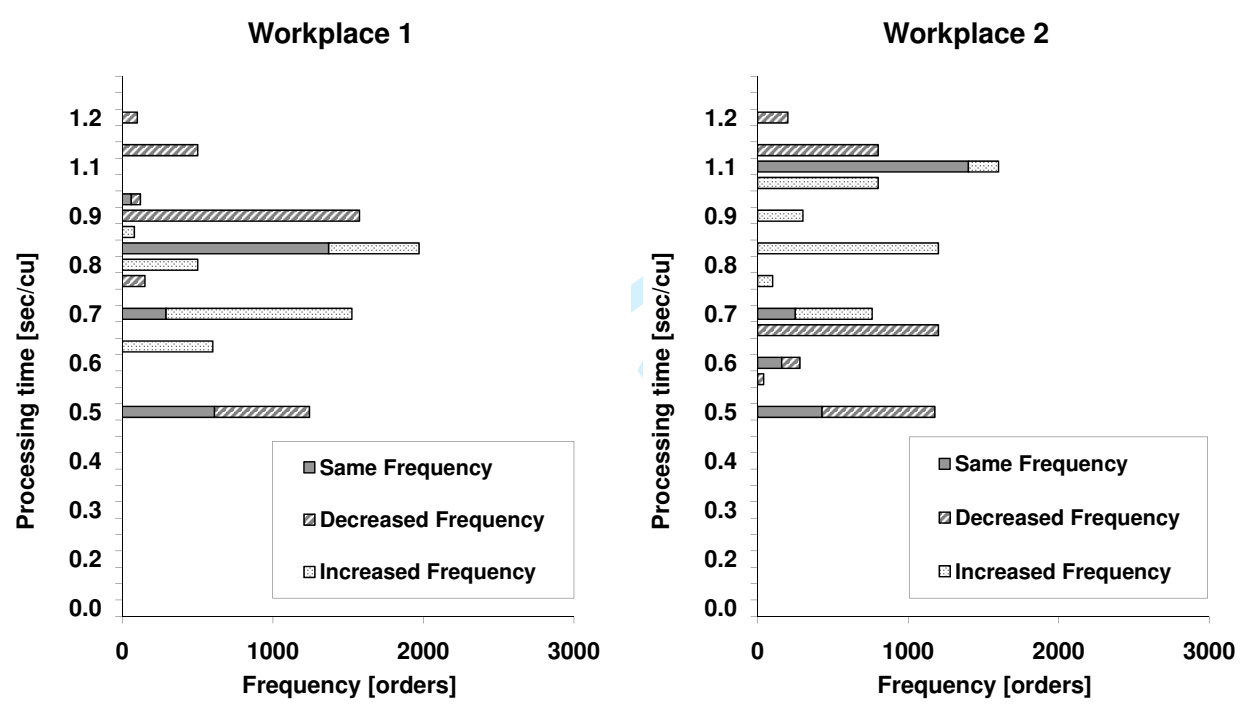

Figure 9.: The two workplaces after the second shift

Besides the above example, seven more pairs of workplaces were analyzed during the first workshop. In total, it was possible to reduce the average linear overloadtime by about $0.185 \frac{\mathrm{sec}}{\mathrm{cu}}$ which is about one fifth of the cycle time. This was possible by shifting 21 tasks only. The relative decrease was about $34 \%$. Furthermore, high workloads, especially those a lot higher than the cycle time, were reduced particularly because the average square overload-time was reduced by about $54 \%$. The detailed results can be seen in Table 3 . Scenario 1 is the example shown above.

The final task assignment was evaluated by simulating the assembly line before and after the reconfiguration. The number of floater assignments could be reduced from 487 to 278 . This meant a decrease of $43 \%$. As a result, the number of needed floaters decreased from 20 to 13.

After the first successful workshop, a second assembly line for a different car model was investigated. In this case it was possible to reduce the linear overload- 


\begin{tabular}{c|r|r|r|r|r|r|r|r|r} 
& \multicolumn{3}{|c|}{$\Delta^{\text {LinPos }}\left(\frac{\text { sec }}{c \mid}\right)$} & \multicolumn{3}{|c|}{$\Delta^{\text {QuadPos }}\left(\frac{\text { sec }^{2}}{\text { Su }^{u}}\right)$} & & moved \\
Scen. & before & after & diff. & diff.[\%] & before & after & diff. & diff.[\%] & tasks \\
\hline 1 & 0,060 & 0,024 & $-0,036$ & $-60 \%$ & 0,532 & 0,149 & $-0,383$ & $-72 \%$ & 2 \\
2 & 0,088 & 0,057 & $-0,031$ & $-36 \%$ & 1,623 & 0,494 & $-1,129$ & $-70 \%$ & 2 \\
3 & 0,034 & 0,012 & $-0,022$ & $-65 \%$ & 0,223 & 0,169 & $-0,055$ & $-24 \%$ & 2 \\
4 & 0,010 & 0,010 & 0,000 & $4 \%$ & 0,423 & 0,094 & $-0,330$ & $-78 \%$ & 4 \\
5 & 0,113 & 0,086 & $-0,026$ & $-24 \%$ & 4,249 & 2,370 & $-1,879$ & $-44 \%$ & 6 \\
6 & 0,051 & 0,051 & 0,001 & $2 \%$ & 1,386 & 1,073 & $-0,313$ & $-23 \%$ & 1 \\
7 & 0,124 & 0,059 & $-0,065$ & $-53 \%$ & 3,248 & 0,780 & $-2,468$ & $-76 \%$ & 2 \\
8 & 0,063 & 0,058 & $-0,005$ & $-8 \%$ & 2,058 & 1,252 & $-0,806$ & $-39 \%$ & 2 \\
\hline$\sum / \oslash$ & 0,542 & 0,357 & $-0,185$ & $-30 \%$ & 13,742 & 6,379 & $-7,363$ & $-53 \%$ & 21
\end{tabular}

Table 3.: Results of the first workshop

time by 24 seconds. That meant a relative decrease of $18 \%$. Additionally, the square overload-time was reduced by about $20 \%$. To achieve these results, only five scenarios and only 23 shifts were needed. The details of the second case can be seen in Table 4.

\begin{tabular}{c|r|r|r|r|r|r|r|r|r} 
& \multicolumn{3}{|c|}{$\Delta^{\text {LinPos }}\left(\frac{s e c}{c u}\right)$} & & \multicolumn{3}{|c|}{$\Delta^{\text {QuadPos }}\left(\frac{\text { sec }^{2}}{c u}\right)$} & & moved \\
Scen. & before & after & diff. & diff.[\%] & before & after & diff. & diff.[\%] & tasks \\
\hline 1 & 0,469 & 0,313 & $-0,156$ & $-33 \%$ & 57,942 & 33,187 & $-24,755$ & $-43 \%$ & 11 \\
2 & 0,115 & 0,044 & $-0,071$ & $-61 \%$ & 2,168 & 1,552 & $-0,616$ & $-28 \%$ & 1 \\
3 & 0,403 & 0,369 & $-0,035$ & $-9 \%$ & 52,860 & 44,373 & $-8,488$ & $-16 \%$ & 5 \\
4 & 0,327 & 0,323 & $-0,004$ & $-1 \%$ & 43,062 & 38,691 & $-4,371$ & $-10 \%$ & 2 \\
5 & 0,406 & 0,365 & $-0,041$ & $-10 \%$ & 40,508 & 39,829 & $-0,678$ & $-2 \%$ & 4 \\
\hline$\sum / \oslash$ & 1,72 & 1,41 & $-0,31$ & $-23 \%$ & 196,54 & 157,63 & $-38,91$ & $-20 \%$ & 21
\end{tabular}

Table 4.: Results for the second test problem

The second case was simulated as well. Here, the number of floater operations decreased from 122 to 84 , a decrease of about $31 \%$. At the same time, the number of needed floaters could be reduced from five to four.

\section{Conclusions and Future Research Directions}

In this paper we considered the mixed model assembly line reconfiguration problem in the context of auto production. We presented a mathematical optimization model which aims at minimizing the costs incurred by overload situations, regular workers and reconfiguration measures. Due to the model's complexity, lack of data and acceptance issues it is hardly possible to fully automate the solution process in an industrial environment. Therefore, we presented a decision support approach which consists of visualization components, new numerical indicators and an integrated optimization procedure to semi-automate the optimization process. In particular, reconfiguration costs can be taken into account and no complete precedence graph is required which is almost impossible to obtain in the final assembly phase of car production. Finally, we showed on the basis of two industrial case studies that our approach can be successfully applied in a practical environment where it was capable to drastically reduce the occurrence of overload situations. Our further research is going to concentrate on the effects on quality of production effected by different reconfiguration solutions. Additionally new methods to obtain a precedence graph automatically in production environments with high product variety are needed. By this it could be guaranteed that the heuristic, proposed in this paper, could be restricted to feasible solutions concerning the technical prece- 
dence restrictions. Nevertheless methods that will be applied in practice will always need the interaction of the planner.

\section{References}

Becker, C. and Scholl, A., 2006. A survey on problems and methods in generalized assembly line balancing. European Journal of Operational Research, 168, 694715.

Becker, C. and Scholl, A., 2009. Balancing assembly lines with variable parallel workplaces: Problem definition and effective solution procedure. European Journal of Operational Research, 199, 359-374.

Becker, H., 2007. Auf Crashkurs - Automobilindustrie im globalen Verdrängungswettbewerb -. Vol. 2. Springer Berlin Heidelberg.

Bock, S., Rosenberg, O. and van Brackel, T., 2006. Controlling mixed-model assembly lines in real-time by using distributed systems. European Journal of Operational Research, 168, 880-904.

Boysen, N., Fliedner, M. and Scholl, A., 2007a. A classification of assembly line balancing problems. European Journal of Operational Research, 183, 674-693.

Boysen, N., Fliedner, M. and Scholl, A., 2007b. Production Planning of MixedModel Assembly Lines: Overview and Extensions. To appear in: Production Planning and Control.

Boysen, N., Fliedner, M. and Scholl, A., 2008. Assembly line balancing: Which model to use when?. International Journal of Production Economics, 111, $509-528$.

Boysen, N., Fliedner, M. and Scholl, A., 2009. Assembly line balancing: Joint precedence graphs under high product variety. IIE Transactions, 41, 183-193.

Bruccoleri, M., Renna, P. and Perrone, G., 2005. Reconfiguration: a key to handle exceptions and performance deteriorations in manufacturing operations. International Journal of Production Research, 43 (19), 4125-4145.

Bukchin, J., Dar-El, E. and Rubinovitz, J., 2002. Mixed model assembly line design in a make-to-order environment. Computers $\& 3$ Industrial Engineering, 41, 405421.

Chiang, W.C., 1998. The application of a tabu search metaheuristic to the assembly line balancing problem. Annals of Operations Research, 77, 2009-227.

Domschke, W., Klein, R. and Scholl, A., 1996. Antizipative Leistungsabstimmung bei moderner Variantenfließfertigung. Zeitschrift für Betriebswirtschaft, 66, $1465-1490$.

Emde, S., Boysen, N. and Scholl, A., 2008. Balancing mixed-model assembly lines: A computational evaluation of objectives to smoothen workload.. To appear in: International Journal of Production Research.

Erel, E., Sabuncuoglu, I. and Sekerci, H., 2005. Stochastic assembly line balancing using beam search. International Journal of Production Research, 43 (7), 14111426 .

Falkenauer, E., 2005. Line Balancing in the Real World. In: Proceedings of the International Conference on Product Lifecycle Management PLM 05, Lumiere University of Lyon, France 2005 (on cd-rom).

Gamberini, R., Grassi, A. and Rimini, B., 2006. A new multi-objective heuristic algorithm for solving the stochastic assembly line re-balancing problem. International Journal of Production Economics, 102, 226-243.

Gottschalk, S., 2006. Dedicated Flexibility - Komplexitätsoptimale Gestaltung manueller Serienmontage. Thesis (PhD). Rheinisch-Westfälische Technische Hochschule Aachen. 
Ho, J.K.L., 2005. A proposed approach for reconfiguration of flexible assembly line systems by motion genes. International Journal of Production Research, 43 (9), 1729-1749.

Hotman, E., 2006. Decision Support Expert System - for Process Selection. Thesis $(\mathrm{PhD})$. Faculty of Electrical Engineering, Computer Science and Mathematics - University of Paderborn.

Kim, Y.K., Y., K. and J., K.Y., 2000. Two-sided assembly line balancing: a genetic algorithm approach. Production Planning and Control, 11 (1), 44-53.

McMullen, P.R. and Frazier, G.V., 1998. Using simulated annealing to solve a multiobjective assembly line balancing problem with parallel workstations. International Journal of Production Research, 10 (10), 2717-2741.

Merengo, C., Nava, F. and Pozzetti, A., 1999. Balancing and sequencing manual mixed-model assembly lines. International Journal of Production Research, 37 (12), 2835-2860.

Meyr, H., 2004. Supply chain planning in the German automotive industry. OR Spectrum, 26, 447-470.

Pohl, A., 1996. Leapfrogging bei technologischen Innovationen: Ein Erklärungsansatz auf Basis der Theorie des wahrgenommenen Risikos. Wiesbaden: Gabler.

Salveson, M., 1955. The assembly line balancing problem. The Journal of Industrial Engineering, 6(3), 18-25.

Scholl, A., Fliedner, M. and Boysen, N., 2009. ABSALOM: Balancing assembly lines with assignment restrictions. To appear in: European Journal of Operational Research.

Scholl, A. and Klein, R., 1997. SALOME: a bidirectional branch and bound procedure for assembly line balancing. INFORMS Journal on Computing, 9, 319334.

Schuh, G., 2006. Produktionsplanung und-steuerung. Springer.

Smith, C., 1998. Computer-Supported Decision Making - Meeting the Decision Demands of Modern Organizations. Ablex Publishing Corporation.

Sprecher, A., 1999. A competitive branch-and-bound algorithm for the simple assembly line balancing problem. International Journal of Production Research, 37 (8), 1787-1816.

Sprecher, A., 2003. Dynamic search tree decomposition for balancing assembly lines by parallel search. International Journal of Production Research, 43 (7), 1413-1430.

Turban, E. and Aronson, J.E., 1998. Decision Support Systems and Intelligent Systems. 5 Prentice-Hall.

Vilarinho, P.M. and Simaria, A.S., 2002. A two-stage heuristic method for balancing mixed-model assembly lines with parallel workstations. International Journal of Production Research, 40 (6), 1405-1420.

Wiendahl, H.P. and Gerst, D., 2004. Variantenbeherrschung in der Montage. Springer. 